\title{
ECODEFECOGRAFIA TRIDIMENSIONAL DINÂMICA. NOVA TÉCNICA PARA AVALIAÇÃO DA SÍNDROME DA DEFECAÇÃO OBSTRUÍDA (SDO)
}

\author{
STHELA MARIA MURAD-REGADAS ${ }^{1}$, FRANCISCO SÉRGIO PINHEIRO REGADAS ${ }^{1}$, LUSMAR VERA \\ RODRIGUES ${ }^{1}$, RODRIGO DORSFELD ESCALANTE ${ }^{1}$, FLAVIO R.S. SILVA ${ }^{1}$, DORYANE M.R. LIMA ${ }^{1}$, FÁBIO ALVES \\ SOARES $^{1}$, ROSILMA GORETE LIMA BARRETO ${ }^{1}$, FRANCISCO SERGIO PINHEIRO REGADAS FILHO ${ }^{1}$
}

${ }^{1}$ Serviço de Colo-proctologia da Hospital das Clínicas da Universidade Federal do Ceará

MURAD-REGADAS SM, REGADAS FSP, RODRIGUES LV, ESCALANTE RD, SILVA FRS, LIMA DMR, SOARES FA, BARRETO RGL, REGADAS FILHO FSP. Ecodefecografia Tridimensional Dinâmica. Nova Técnica para Avaliação da Síndrome da Defecação Obstruída (SDO). Rev bras Coloproct, 2006;26(2):168-177.

RESUMO: O objetivo deste estudo é apresentar novas técnicas para avaliação da SDO, utilizando a ultra-sonografia endorretal tridimensional dinâmica e comparando os resultados com a defecografia. Foram incluídas neste estudo 25 mulheres adultas, distribuídas em dois grupos. Grupo I: 15 mulheres normais, idade média de 52,4 anos (23-76) e todas se submeteram ao exame proctológico completo e à ultra-sonografia anorretal tri-dimensional dinâmica para se estabelecer os padrões de normalidade do canal anal e reto. Grupo II: 10 pacientes mulheres com evacuação obstruída, idade média de 47,8 anos (33 a 65 anos), apresentando como principais sintomas a sensação de evacuação incompleta, disquezia e digitação vaginal ou perineal. Submeteram-se a exame proctológico completo, seguindo-se defecografia e posteriormente ecodefecografia por dois examinadores que desconheciam o resultado do exame anterior. A ecodefecografia dinâmica foi realizada com um equipamento B-K Medical ${ }^{\circledR}$, sonda $360^{\circ}$, tipo 2050 , com escaneamento automático durante 50 segundos com $6 \mathrm{~cm}$ de extensão. $O$ tamanho médio do ângulo formado pelo músculo PR no repouso foi 87,13 (variação 78,9 - 90,8 $\mathbf{8}^{\circ}( \pm 1,01)$ e no esforço evacuatório 99,22 ${ }^{\circ}$ (variação 84,9 - 114,5 ${ }^{\circ}( \pm 1,84)$ nas mulheres normais (grupo I). Houve elevação do ângulo em todas as pacientes normais, significando relaxamento normal do PR durante o esforço evacuatório. Com relação à avaliação da anoretocele, a parede posterior da vagina se manteve na posição horizontal durante todo o esforço evacuatório, exceto nas portadoras de anoretocele. Foram diagnosticadas anoretocele (grau $I=1$, grau $I I=5$, grau III = 4) em todas as pacientes do grupo II pelo exame clínico e defecografia. Todos os casos foram confirmados pela ecodefecografia. A partir destes resultados, foram estabelecidos os valores para classificar a anorectocele de acordo com a ecodefecografia (grau I - distância entre as posições da parede vaginal até $5,0 \mathrm{~mm}$, grau II de 6,0 a $12,0 \mathrm{~mm}$, grau III além de 12,0mm). Foi identificado anismus em uma paciente com anoretocele grau II e em outra com grau III na defecografia e confirmado na ecodefecografia pela redução no ângulo formado pelo PR ao comparar as posições em repouso e durante o esforço evacuatório. A defecografia demonstrou também quatro casos de intussuscepção enquanto a ecodefecografia confirmou estes casos e identificou dois outros. Em conclusão, a ecodefecografia pode ser utilizada como um método alternativo para o diagnóstico da SDO, pois identifica e quantifica todas as disfunções anorretais responsáveis pela evacuação obstruída. Apresenta também a grande vantagem de avaliar os distúrbios da continência, identificando lesões esfincterianas, mesmo ocultas. É minimamente invasivo, bem tolerado, baixo custo, não expondo o paciente à radiação e demonstrando com precisão todas as estruturas anatômicas envolvidas com a defecação.

Descritores: Evacuação Obstruída, Defecografia, Ultra-sonografia, Reto, Ânus, Retocele, Anismus, Intussuscepção retal.

\footnotetext{
Trabalho realizado no Serviço de Colo-proctologia da Hospital das Clínicas da Universidade Federal do Ceará e Centro de Colo-proctologia do Ceará
}

$\overline{\text { Recebido em } 08 / 06 / 2006}$

Aceito para publicação em 27/06/2006 
Rev bras Coloproct Abril/Junho, 2006

\section{INTRODUÇÃO}

Os distúrbios da evacuação resultam em alterações anatômicas e funcionais em múltiplos compartimentos pélvicos, produzindo a retocele, intussuscepção, enterocele, descenso perineal, ausência de relaxamento esfincteriano ou contração paradoxal da musculatura esfincteriana voluntária (anismus).

A defecografia é o método mais comumente utilizado para avaliar as desordens anorretais funcionais. Estudos demonstram a eficácia desta técnica no diagnóstico da anorretocele, contração paradoxal do músculo puborretal, intussuscepção retal e enterocele ${ }^{1-8}$. É um exame minimamente invasivo, seguro e tecnicamente simples. Contudo, existe a desvantagem de não demonstrar claramente as estruturas anatômicas envolvidas, é desconfortável, sobretudo para os mais idosos e expõe o paciente à radiação.

Tem sido demonstrada a aplicação da ultrasonografia dinâmica ${ }^{9-12}$ e a ressonância nuclear magnética dinâmica ${ }^{13-15}$ na avaliação da Síndrome da Defecação Obstruída (SDO), com resultados bastante satisfatórios. As técnicas desenvolvidas com a ultrasonografia dinâmica utilizam tipos diferentes de transdutores. Barthet e cols. ${ }^{9}$ utilizaram um transdutor trans-retal linear, demonstrando resultados semelhantes à defecografia enquanto Beer-Gabel e cols. ${ }^{10,11}$ desenvolveram a técnica com o transdutor transperineal, utilizando gel intra-retal e intra-vaginal, demonstrando também resultados semelhantes à defecografia. Van Outryve e cols. ${ }^{12}$ avaliaram exclusivamente pacientes portadores de anismus com transdutor linear ano-retal. Utilizaram as medições de comprimento e espessura da musculatura esfincteriana para determinar o relaxamento e a contração muscular durante o esforço evacuatório.

O objetivo deste estudo é apresentar novas técnicas para avaliação da SDO, utilizando a ultrasonografia anorretal tridimensional dinâmica (ecodefecografia) e comparando os resultados com a defecografia.

\section{PACIENTES E MÉTODOS}

Foram incluídas neste estudo 25 mulheres adultas, sendo 15 normais (Grupo I) e 10 com quadro clínico de evacuação obstruída (Grupo II).

Grupo I : Apresentaram idade média de 52,4 anos (variação 23-76) e cinco haviam sido submetidas a pelo menos um parto vaginal. Todas se submeteram a exame proctológico completo e a ultra-sonografia anorretal tri-dimensional dinâmica com o objetivo de se estabelecer os padrões de normalidade do canal anal e reto

Grupo II: Apresentaram idade média de 47,8 anos (variação entre 33 a 65 anos) e os principais sintomas referidos foram sensação de evacuação incompleta, disquezia e digitação vaginal ou perineal. Dessas, nove pacientes foram submetidas previamente a pelo menos um parto vaginal. Submeteram-se a exame proctológico completo, seguindo-se defecografia e posteriormente a ultra-sonografia anorretal tridimensional dinâmica (ecodefecografia) por dois examinadores que desconheciam o resultado do exame anterior.

A anoretocele foi classificada pelo exame clínico em grau I (pequena distensão da parede anterior do canal anal superior durante o toque retal), grau II (distensão até o intróito vaginal), grau III (ultrapassando o intróito vaginal) e pela defecografia (grau $\mathrm{I}=$ até $2,0 \mathrm{~cm}$, grau II $2,0-4,0 \mathrm{~cm}$, grau III $>4,0 \mathrm{~cm}$ ).

A ecodefecografia dinâmica foi realizada com um equipamento B-K Medical ${ }^{\circledR}$, com sonda $360^{\circ}$, tipo 2050 com escaneamento automático com $6 \mathrm{~cm}$ de extensão e 50 segundos de duração. $\mathrm{O}$ exame consiste na captura de uma seqüencia de imagens axiais $(0,25 \mathrm{~mm})$ que resulta num cubo 3-D de imagem, sendo gravada em tempo real que possibilita a análise em múltiplos planos. Todas as pacientes concordaram em participar deste protocolo prospectivo através de consentimento informado.

Técnica da Defecografia - Os dados foram obtidos mediante evacuação de pasta baritada com as pacientes sentadas em assento radio-transparente e sob radioscopia pélvica de incidência horizontal em perfil. A pasta baritada tem consistência padronizada, de modo a aproximar-se da consistência fecal e é introduzida no reto em volume igual a $300 \mathrm{ml}$ ou até produzir vontade evacuatória. Os dados são avaliados pela captura digital (fase dinâmica) e filmes convencionais (fase estática), sendo aferidos parâmetros, como mobilidade do puborretal à evacuação; alargamento da distância retovaginal; presença de anorretocele e 
Rev bras Coloproct Abril/Junho, 2006 retenção de contraste na mesma; resíduo contrastado ao fim da evacuação; alterações de projeção da mucosa retal sugestivas de intussuscepção; compressão extrínseca sugestiva de enterocele ou sigmoidocele; ângulo anorretal (em graus); posição da junção anorretal (em centímetros); descenso perineal (em centímetros).

\section{TÉCNICA DA ECODEFECOGRAFIA}

É necessário preparo prévio com clister retal duas horas antes do exame. $\mathrm{O}$ transdutor é introduzido no reto e mantido fixo, entre 6 a $7 \mathrm{~cm}$ da margem anal. As imagens para avaliar todas as alterações funcionais da defecação são obtidas em três escaneamentos automáticos para posterior análise nos planos axial e longitudinal. Cada escaneamento tem a duração de 50 segundos.

Escaneamento 1- O probe é posicionado a 6 $\mathrm{cm}$ da margem anal. Segue o escaneamento do reto inferior, junção ano-retal e canal anal com o paciente em repouso. É avaliada a conformação anatômica do canal anal, buscando identificar eventuais lesões musculares, mesmo em pacientes assintomáticos (lesões ocultas)

Escaneamento 2 - O probe é posicionado a $7 \mathrm{~cm}$ da margem anal e segue outra seqüência de repouso e esforço evacuatório no mesmo escaneamento. É iniciado com repouso durante 15 segundos, capturando imagens do reto inferior. Segue-se o esforço evacuatório durante 20 segundos, correspondendo ao escaneamento da junção ano-retal, canal anal superiormédio proximal, parede posterior da vagina e bexiga. Finalmente, mantém-se em repouso durante os 15 segundos finais, correspondendo ao canal anal médio distal e inferior.

Escaneamento 3 - Injetam-se inicialmente 120 $\mathrm{ml}$ de gel ultra-sônico na ampola retal. O probe é posicionado a $7 \mathrm{~cm}$ da margem anal e segue a mesma técnica utilizada no escaneamento 2 . No entanto, esse escaneamento deve ser repetido sempre quando necessário nos casos de dúvida com relação à qualidade da imagem obtida. Pode ser necessário aplicar mais gel intra-retal.
É importante também certificar-se que o paciente está efetivamente realizando o esforço evacuatório com o transdutor posicionado no canal anal e reto.

Interpretação das imagens - Após concluídos os escaneamentos supra-citados, as imagens dinâmicas (esforço evacuatório) foram analisadas, comparandoas com a posição das estruturas anatômicas envolvidas com a defecação na posição de repouso utilizando linhas e/ou ângulos para medições de referência. As imagens foram analisadas estabelecendo-se os padrões de normalidade ocorridos durante o esforço evacuatório e procurando identificar e quantificar as alterações anátomo-funcionais presentes nos pacientes com evacuação obstruída.

1 - Ecodefecografia normal (projeção longitudinal mediana) - A parede posterior da vagina empurra o reto e o canal anal superior para baixo e para trás, mantendo-se em linha reta na posição horizontal durante o esforço evacuatório (Figura-1)

2- Anismus- (Plano longitudinal mediano) A referência utilizada para pesquisa do anismus foi o músculo puborretal (PR). Determinou-se um ângulo formado pela confluência de uma linha traçada paralela à borda interna do PR $(1,5 \mathrm{~cm})$ com uma outra linha vertical, longitudinal ao eixo do canal anal. Esse ângulo foi calculado no repouso (escaneamento 1) e no esforço evacuatório (escaneamentos 2 e 3 ). O exame foi considerado normal quando ocorria aumento deste ângulo devido aumentar a distância entre o transdutor e o PR pelo relaxamento do EAE e PR durante o esforço evacuatório (Figura-2a,b). Ao contrário, quando havia contração paradoxal do EAE e PR durante o esforço evacuatório, ocorria redução do ângulo devido à diminuição da distância entre o endoprobe e o PR. (Figura-3)

2- Anoretocele - (projeção longitudinal mediano) - A anorretocele foi identificada traçando duas linhas horizontais paralelas na parede posterior da vagina durante o esforço evacuatório, uma na posição inicial quando a vagina empurra para baixo o reto, e outra no ponto de maior distensão anterior da junção anorretal e canal anal superior, fazendo protrusão para a luz vaginal (Figuras 4). A distância 


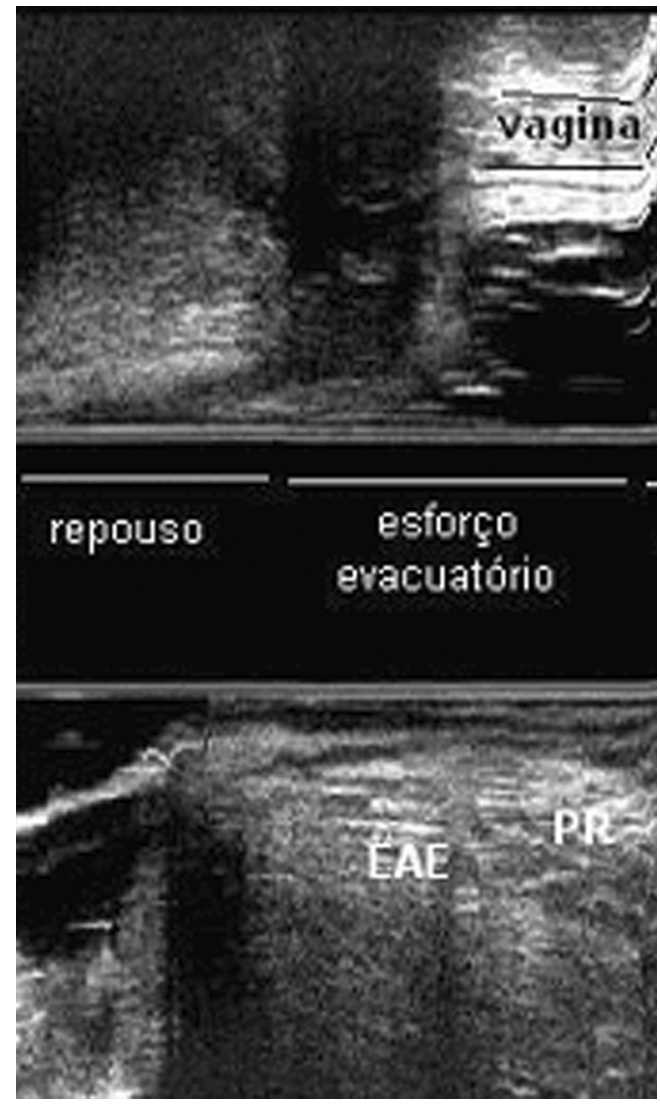

Figura 1 - Ecodefecografia em paciente normal. Plano longitudinal mediano (LM). A parede da vagina é mantida na posição horizontal durante esforço evacuatório. EAE- Esfincter Anal Externo / PR-Puborretal

entre as diferentes posições da parede posterior da vagina durante o esforço evacuatório caracteriza os diferentes tamanhos da anorretocele (Figura 5). E a quantificação do tamanho à retocele na ecodefecografia foi estabelecido de acordo com o exame clínico e a defecografia.

3- Intussuscepção - Foi identificada claramente pela imagem de duas camadas musculares paralelas durante o esforço evacuatório sem gel intraretal nos planos axial e longitudinal (escaneamentos 2) (Figura 6 a). Quando se utilizou gel intra-retal (escaneamento 3), identificaram-se as camadas da parede retal projetando-se para a luz do reto (Figura 6 b). No entanto, as intussuscepções menores (ocultas) caracterizam-se por pequenos deslocamentos, mantendo as camadas retais quase paralelas entre si, sendo necessário associar os escaneamentos com e sem

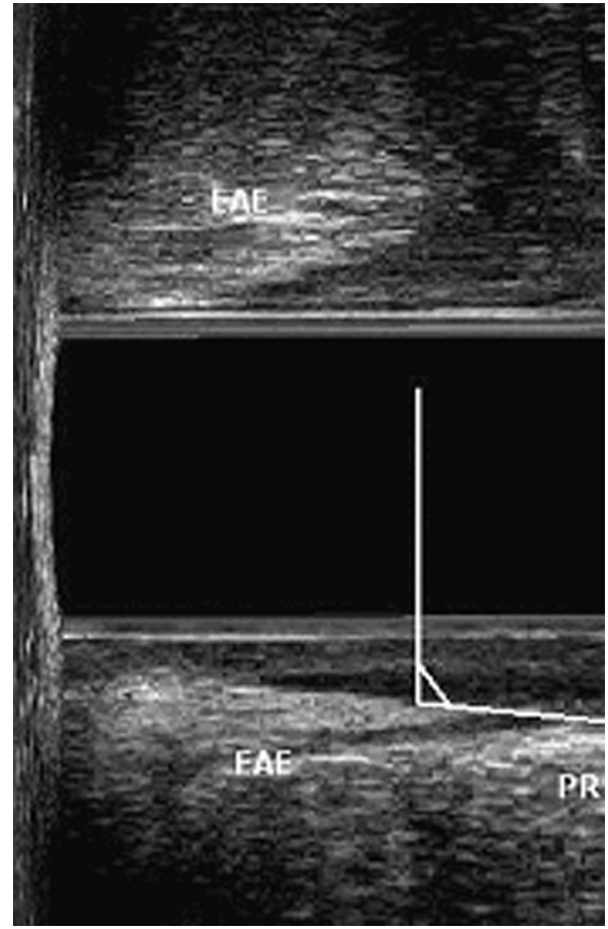

A

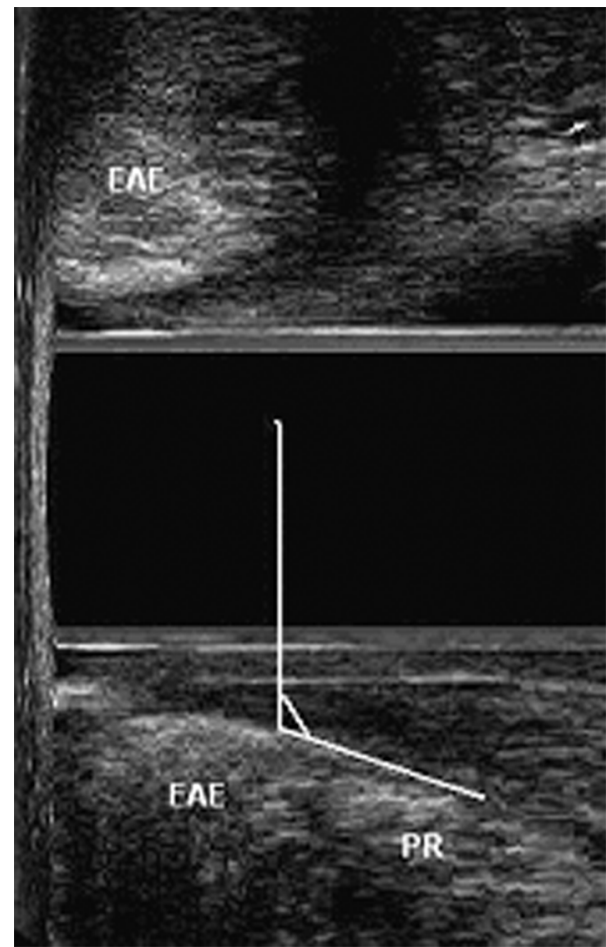

B

Figura 2 - Paciente Normal. Ângulo formado pela confluência de uma linha traçada paralela à borda interna do $P R(1,5 \mathrm{~cm}) \mathrm{com}$ uma outra linha vertical, perpendicular ao eixo do canal anal. Plano LM. a - Repous. b - Esforço Evacuatório - Aumento no Ângulo 


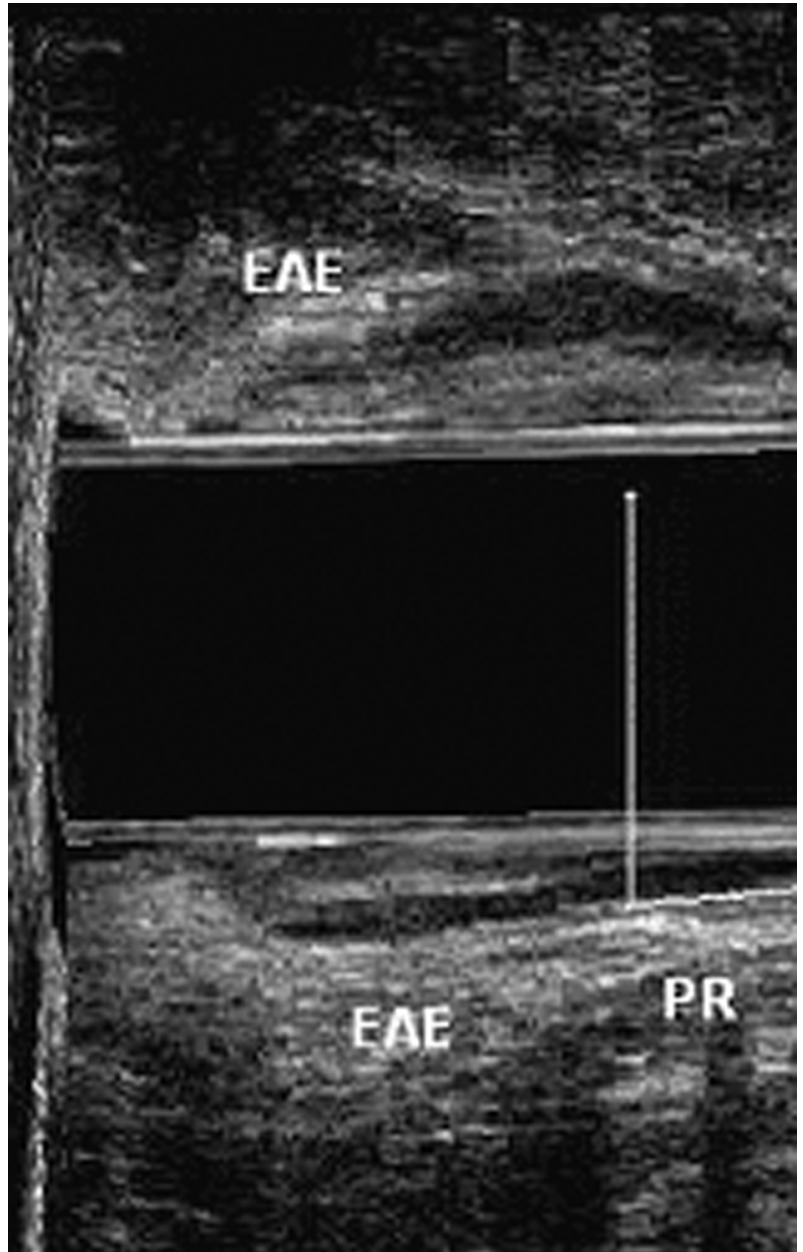

Figura 3 - Paciente com Anismus. Plano LM. Há redução no ângulo do puborretal durante o esforço evacuatório.

gel intra-retal, nas projeções axial e longitudinal. Já nas maiores, o deslocamento das camadas da parede do reto é mais pronunciado, projetando-se de forma perpendicular entre si (Figura 7).

\section{4- Ano-retocele com Intussuscepção-}

Identifica-se normalmente a presença de intussuscepção retal associada a ano-retoceles volumosas e este processo é claramente demonstrado em imagens obtidas pela ecodefecografia no local da herniação. Nas intussuscepções grandes, as camadas musculares da parede do reto são identificadas ao nível da junção anorretal e canal anal superior, no local onde ocorre maior herniação.

5- Enterocele - As alças intestinais foram normalmente visualizadas na projeção do quadrante

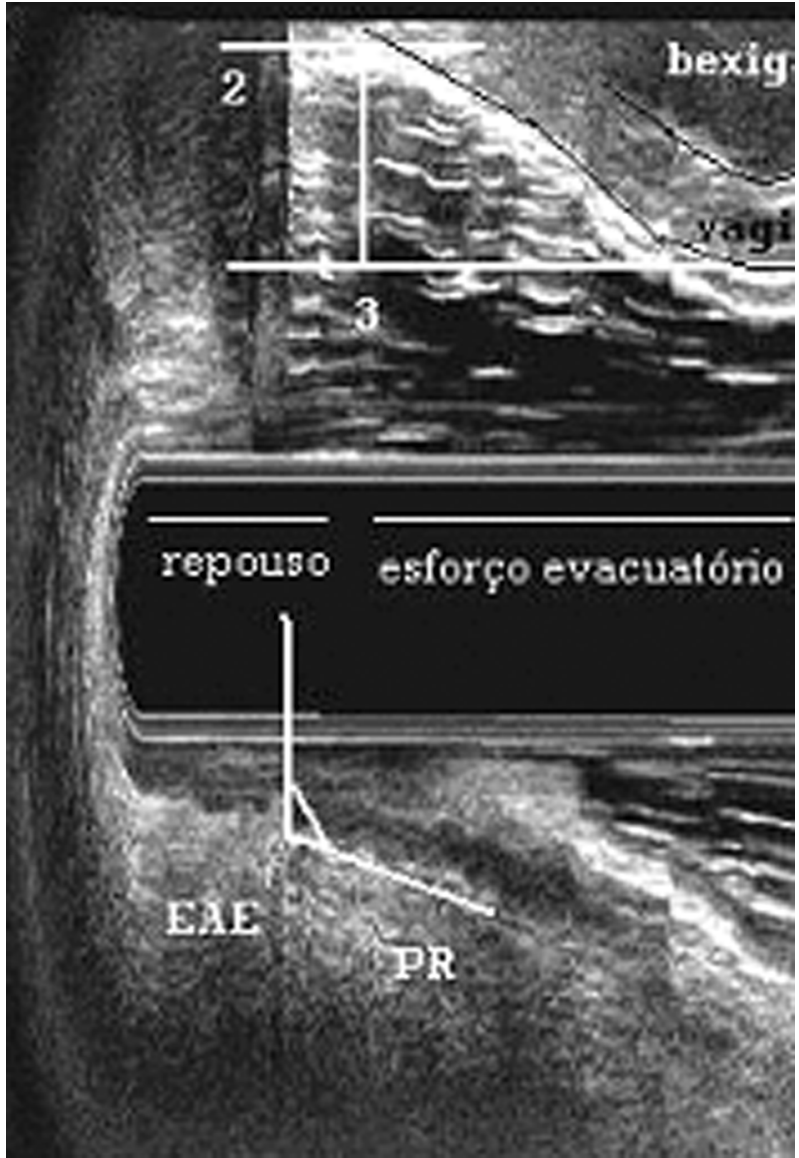

Figura 4-Anoretocele. Plano LM

Linha 1- Paralela a parede posterior da vagina no início do esforço evacuatóri.

Linha 2 - Paralela a parede posterior da vagina no ponto de maior herniação.

Linha 3 - Distância entre as linhas - Classificação das anoretoceles. Ângulo do puborretal em relaxamento no esforço evacuatório.

anterior do reto médio-inferior, proximal à bexiga e ao útero, mesmo durante o esforço evacuatório. A enterocele caracteriza-se pela identificação de alças intestinais na projeção do reto inferior, ao nível do músculo pubo-retal e podem ser claramente visualizadas nos planos axial e longitudinal (escaneamentos 2,3)

Todos os dados obtidos nas medições do ângulo formado pelo músculo PR durante o esforço evacuatório no grupo I foram analisados utilizando o test $\mathrm{t}$ Student. Os valores foram expressos em média, seguidos do erro padrão. 


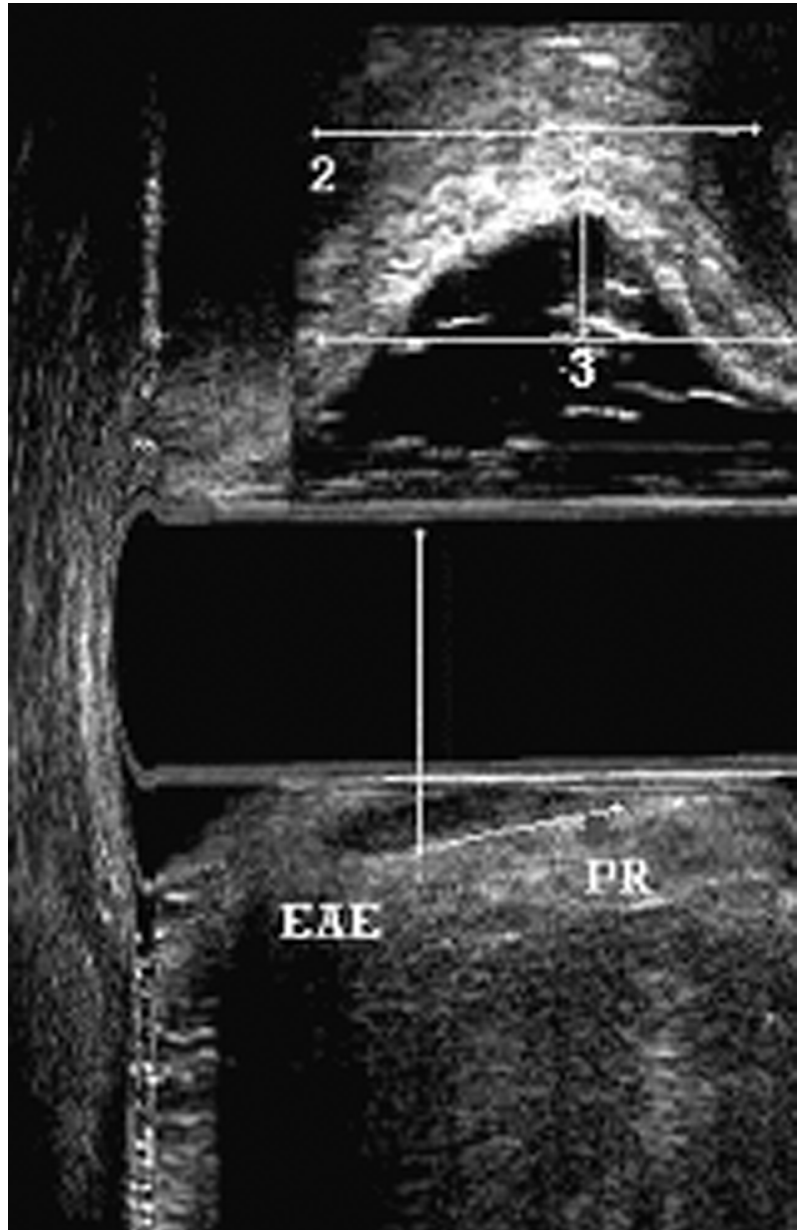

Figura 5 - Anoretocele ( Plano LM).

Ângulo do puborretal determina ausência de relaxamento muscular (Anismus)

\section{RESULTADOS}

\section{GRUPO I (MULHERES NORMAIS)}

Avaliação do relaxamento do músculo Puborretal - A média do ângulo formado pelo músculo PR no repouso foi $87,13^{\circ}$ (variação $\left.78,9-90,8^{\circ}\right)( \pm 1,01)$ e no esforço evacuatório $99,22^{\circ}$ (variação $\left.84,9-114,5^{\circ}\right)( \pm 1,84)$. Houve elevação do ângulo em todos os pacientes normais examinados, significando relaxamento do PR durante o esforço evacuatório.

Avaliação da Anoretocele- A parede posterior da vagina se manteve na posição horizontal durante todo o esforço evacuatório, exceto nas pacientes portadoras de anoretocele.
A

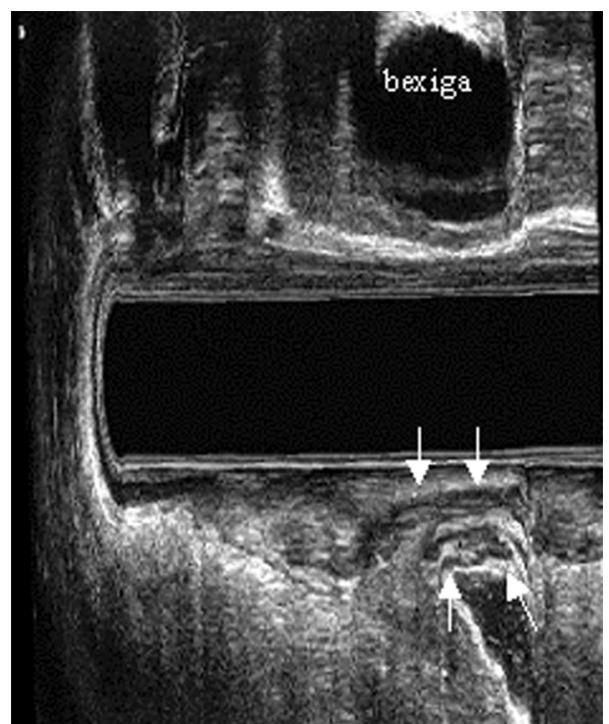

B

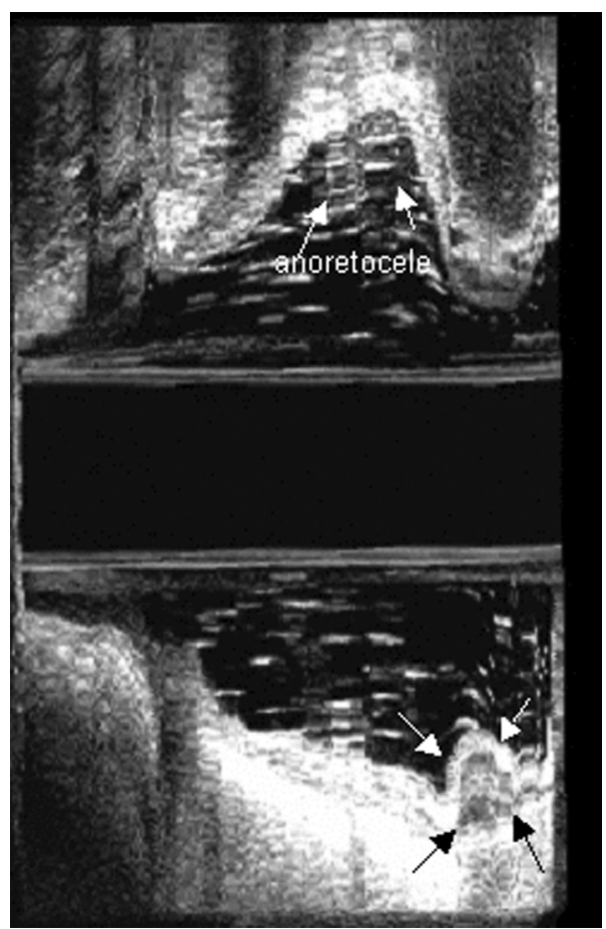

Figura 6 - Intussuscepção visualizada no quadrante posterior. ( Duas camadas musculares própria sobrepostas). Plano longitudinal com diagonal.

a-Escaneamento sem gel

b- Escaneamento com gel foi identificado também anoretocele 


\section{GRUPO II (PACIENTES COM EVACUAÇÃO OBSTRUÍDA)}

Foram diagnosticadas anoretoceles (grau I $=1$, grau $\mathrm{II}=5$, grau $\mathrm{III}=4$ ) em todas as pacientes pelo exame clínico e defecografia. Os mesmos resultados foram identificados na ecodefecografia e, baseando-se na classificação clínica e defecográfica, estabeleceram-se os valores referentes aos diversos tamanhos da anoretocele. Estabeleceu-se que a distância de até $5,0 \mathrm{~mm}$ entre a posição da parede posterior da vagina durante o esforço evacuatório corresponde ao grau I, entre 6,0 a $12,0 \mathrm{~mm}$ ao grau II e além de $12,0 \mathrm{~mm}$ ao grau III (Tabela-1).

Foi identificado anismus em uma paciente com anoretocele grau II e em outra com grau III na defecografia e confirmado na ecodefecografia pela redução no ângulo formado pelo músculo puborretal ao comparar as posições em repouso e durante o esforço evacuatório.

A defecografia demonstrou também quatro casos de intussuscepção, enquanto a ecodefecografia confirmou estes casos e identificou dois outros (Tabela2).

\section{DISCUSSÃO}

A ultra-sonografia anorretal tridimensional dinâmica (ecodefecografia) possibilita avaliação estática e dinâmica da anatomia anorretal e do assoalho pélvico, identificando as estruturas anatômicas envolvidas no mecanismo da evacuação. A principal vantagem é identificar claramente as diferentes posições das estruturas anatômicas anorretais envolvidas na defecação, demonstrando também todas as disfunções responsáveis pela SDO, tais como a anoretocele, intussuscepção, o prolapso mucoso interno, a enterocele e o anismus, sem expor a paciente à radiação. $\mathrm{O}$ exame com a modalidade 3-D com escaneamento automático é simples e nãoinvasivo, pois não necessita mover o transdutor durante o escaneamento e as imagens são adquiridas e gravadas para serem analisadas no próprio equipamento em tempo real após a retirada do endoprobe. Por esta razão, as imagens apresentamse com elevada resolução espacial, sendo possível identificar o início e o término do esforço evacuatório sem produzir distorções anatômicas. É bem tolerado pelos pacientes e realizado no tempo médio entre 5 a 10 minutos. A técnica da ecodefecografia visa simular a evacuação mesmo com o paciente em decúbito lateral esquerdo. Foram introduzidos $120 \mathrm{ml}$ de gel ultra-sônico no reto. Mesmo com consistência diferente das fezes, esse volume foi suficiente para simular a defecação, pois é suficiente para despertar o desejo evacuatório e distender completamente as paredes do reto e canal anal superior durante o esforço evacuatório. Barthet et $\mathrm{al}^{9}$ propuseram uma técnica endosonográfica dinâmica que usa uma sonda longitudinal e utilizaram $50 \mathrm{ml}$ de água dentro do reto para contraste da imagem. Beer-Gabel et al ${ }^{10}$ também relataram resultados satisfatórios na identificação da retocele usando a técnica transperineal e inserindo gel ultra-sônico dentro do reto e da vagina. Tem sido sugerido que a posição em decúbito lateral esquerdo e a sonda no reto, durante o esforço evacuatório, possam resultar em limitação dos amplos movimentos do assoalho pélvico e dificultar a evacuação. No entanto, tal fato não foi observado no presente estudo, pois os pacientes usualmente realizaram o esforço evacuatório adequado e evacuaram o gel ultra-sônico parcial ou totalmente, o que possibilitou identificar e quantificar todas as

Tabela 1 - Graus da Anoretocele- Defecografia versus Ecodefecografia

\begin{tabular}{lccc}
\hline No. Pacientes & Grau da Retocele & Defecografia $(\mathbf{c m})$ & Ecodefecografia $(\mathbf{m m})$ \\
\hline 1 & I & até 2.0 & até 5.0 \\
5 & II & $2.0-4.0$ & $6.0-12.0$ \\
4 & III & $>4.0$ & $>12.0$ \\
\hline
\end{tabular}


Tabela 2 - Achados da Defecografia versus Ecodefecografia

\begin{tabular}{lcc}
\hline & Defecografia & Ecodefecografia \\
\hline Anoretocele & 10 & 10 \\
Anismus & 2 & 2 \\
Intussuscepção Retal & 4 & 6 \\
\hline
\end{tabular}

disfunções da defecação, confirmando todas aquelas previamente identificadas pela defecografia. Outros estudos que utilizam US dinâmica trans-retal e transperineal demonstraram resultados semelhantes à defecografia ${ }^{9,10}$. Este estudo envolveu também 15 mulheres normais para estabelecer os padrões de normalidade na ecodefecografia. A vagina foi considerada como a estrutura anatômica de referência na avaliação da anoretocele devido a sua localização próxima e paralela à parede anterior do canal anal e do reto, seguindo os mesmos movimentos durante a evacuação. Por esta razão, o escaneamento automático deve iniciar com o paciente em repouso (15 seg), seguido pelo esforço evacuatório durante 20 segundos e finalizando com o paciente em repouso (15 seg) novamente para avaliar o intervalo do esforço evacuatório entre os dois repouso. Assim, o movimento da junção anorretal e do canal anal superior produzido durante o esforço evacuatório é completamente visualizado pela imagem ultra-sonográfica de elevada resolução espacial (plano longitudinal mediano). A vagina e a parede anterior do reto e canal anal superior movem-se para baixo e para trás durante o esforço evacuatório, sendo mantidos no plano horizontal nos pacientes normais. Já na anoretocele, ocorre distensão da parede anterior do reto inferior e canal anal superior em direção à vagina durante os 20 segundos do esforço evacuatório. A ano-retocele forma-se inicialmente no canal anal superior e não na parede do reto, como demonstrado em estudos anteriores por Regadas e cols. ${ }^{17}$. O tamanho da ano-retocele é determinado pela medida da distância da parede da vagina entre o inicio e o término do esforço evacuatório. Nas ano-retoceles muito grandes, a parede posterior da vagina não é visualizada na área do escaneamento por causa da limitação da distância focal da sonda. No entanto, mesmo sem identificar a vagina, a anoretocele é classificada como grau III, pois a distância é superior a $12,0 \mathrm{~mm}$.
O tempo de esforço evacuatório, mantido em 20 segundos previamente estabelecido, foi bem tolerado pelas pacientes e suficiente para identificar todas as alterações da defecação.

A avaliação do relaxamento ou contração paradoxal do puborretal no esforço evacuatório é bem definida, medindo o ângulo formado pela confluência de uma linha paralela à borda interna do PR com outra vertical e perpendicular ao eixo do canal anal. $O$ ângulo aumenta no relaxamento do músculo PR nos pacientes normais e reduz naqueles com contração paradoxal do EAE e PR durante o esforço evacuatório. Pode ser avaliado nos escaneamentos sem e com gel.

Com relação à identificação da intussuscepção, é necessário avaliar nos dois escaneamentos. As grandes intussuscepções são facilmente identificadas no escaneamento com gel, pois as camadas da parede retal projetam-se para o interior do reto e do canal anal superior. Já as menores são identificadas associandose os dois tipos de escaneamento. Devido à clareza das imagens obtidas na ecodefecografia, foi identificada intussuscepção em duas pacientes não demonstradas previamente pela defecografia.

A enterocele é facilmente diagnosticada pela visualização de alça intestinal posicionada no espaço reto-vaginal na projeção do músculo PR.

Em conclusão, a ecodefecografia 3-D dinâmica pode ser utilizada como um método alternativo para o diagnóstico da SDO, pois identifica e quantifica todas as disfunções anorretais responsáveis pela evacuação obstruída. Apresenta também a grande vantagem de avaliar os distúrbios da continência, identificando lesões esfincterianas, mesmo ocultas. É minimamente invasivo, bem tolerado, baixo custo, não expondo o paciente à radiação e demonstrando com precisão todas as estruturas anatômicas envolvidas na defecação. 
Rev bras Coloproct Abril/Junho, 2006
Ecodefecografia Tridimensional Dinâmica. Nova Técnica para Avaliação da Síndrome da Defecação Obstruída (SDO)

Sthela Maria Murad-Regadas e Cols.
Vol. 26

SUMMARY: The aim of the present study is to show novel techniques to evaluate the ODS using dynamic three-dimensional endorectal ultrasound and comparing the results with defecography. Twenty-five adult women were included and distributed in two groups. Group I: Fifteen normal women, mean age 52,4 years old (range 23-76) submitted to full proctologic exam and dynamic 3D anorectal ultrasonography to establish the normal patterns of the rectum and anal canal. Group II: Ten female patients complaining of obstructed defecation, mean age 47,8 years old (33 a 65). The main symptoms were incomplete evacuation, disquezia and vaginal or perineal digitation. They were submitted to full proctologic evaluation, followed by defecography and lastly echodefecography by two examiners without knowing the defecography results. The dynamic echodefecography was performed using B-K Medical® equipment, $360^{\circ}$ endoprobe, type 2050, with automatic scan during 50 seconds. The mean angle size formed by the PR at rest position (group I) was $87,13^{\circ}$ (range $\left.78,9-90,8^{\circ}\right)( \pm 1,01)$ and $99,22^{\circ}$ (range $\left.84,9-114,5^{\circ}\right)( \pm 1,84)$ during evacuatory effort.. It was observed that the angle size increased in all normal women, demonstrating PR relaxation during the evacuatory effort. Concerning to anorectocele evaluation, the posterior vagina wall was kept at horizontal position during the evacuatory effort, except in patients with anorectocele. All patients from group II had anorectocele ( grade $I=1$, grade $I I=5$, grade $I I I=4$ ) demonstrated at clinical and defecography evaluation. All cases were also confirmed by echodefecography. Based on such results, it was established the reference parameters to classify anorectocele according to echodefecography. (grade I - distance of the vaginal wall positions until 5,0mm, grade II from 6,0 to 12,0mm, grade III above 12,0mm). Defecography identified anismus in one patient with anorectocele grade II and in another with grade III and both were confirmed at echodefecography by decreasing the PR angle when compared the resting with straining positions. Defecography showed also four cases of rectal intussusceptions while echodefecography confirmed such findings and identified two other cases. In conclusion, echodefecography can be used as an alternative method to assess patients with ODS because it identifies and quantifies all the anorectal dysfunctions associated with the obstructed defecation, with the advantage to evaluate also the continence disturbances, identifying sphincter injuries. It's minimally invasive, well tolerated, low cost, no radiation exposure and demonstrates precisely all the anatomic structures involved with defecation.

Key words: Obstructed Defecation, Defecography, Ultrasonography, Rectum, Anus, Rectocele, Rectal Intussusception

\section{REFERÊNCIAS BIBLIOGRÁFICAS}

1. Felt-Bersma RJ, Luth WJ, Janssen JJ, Meuwissen SG . Defecography in patients with anorectal disorders. Wich findings are clinically relevant? Dis Colon Rectum 1990; 33:277-284.

2. Mellgreen A, Bremmer S, Johansson C et al . Defecography: results of investigations in 2816 patients. Dis Colon Rectum 1994;37:1133-1141.

3. Kelvin FM, Hale DS, Maglinte DD, Patten BJ, Benson JT . Female pelvic organ prolapse: diagnostic contribution of dynamic cystoproctography and comparison with physical examination. ARJ Am J Roentgenol 1999;173:31-37.

4. Karibom U, Nilsson S, Pahlman L, Graf W Defecographic Study of Rectal Evacuation in Constipated Patients and Control Subjects. Radiology 1999;210:103-108

5. Chen HH, Iroatulam A, Alabaz O, Weiss EG, Nogueras JJ, Wexner SD Associations of defecography and physiologic findings in male patients with rectocele. Tech Coloproctol 2001;5:157-161

6. Johansson C, Nilsson BY, Holmstrom B, Dolk A, Mellgren A Association between rectocele and paradoxical sphincter response. Dis Colon Rectum 1992;35:503-509.

7. Siproudhis L, Dautreme S, ropert A et al Dyschezia and rectocele - a marriage of convenience. Hysiologic evaluation of rectocele in a group of 52 women complaining of difficulty in evacuation. Dis Colon Rectum 1993;36:1030-1036

8. Pescatori M and Quondamcarlo CA A new grading of rectal internal mucosal prolapse and its correlation with diagnosis and treatment. Int J Colorectal Dis 1999;14:245-9.

9. Barthet M, Portier F, Heyries L, et al Dynamic Anal Endosonography May Challenge Defecography for Assessing Dynamic Anorectal Disorders: Results of a Prospective Pilot Study. Endoscopy 2000;32(4):300-5.

10. Beer-Gabel M, Teshler M, Barzilai N, et al. Dynamic transperineal ultrasound (DTP-US)- a new method for disgnosis of pelvic floor disorders: techinical details and preliminary results. Dis Colon Rectum 2000;45:239-48.

11. Beer-Gabel M, Teshler M, Schechtman E, Zbar AP. Dynamic transperineal ultrasound vs. defecography in patients with evacuatory difficulty: a pilot study. Int J Colorectal Dis, 2004; 19:60-67.

12. Van Outryve SM, Van Outryve MJ, De Winter BY, Pelckmans PA Is anorectal endosonography valuable in dyschesia? Gut 2002;51(5):695-700.

13. Lienemann A, Anthuber C, Baron A, et al. Dynamic MR colpocystorectography assessing pelvic-floor descent. Eur Radiol 1997;7:1309-17. 
14. Gufler H, Laubengerger J, De Gregorio G, et al Pelvic Floor Descent: dynamic MR imaging using a half-fourier RARE sequence. J Magn Reson Imaging 1999;9:378-83.

15. Kelvin FM, Maglinte DDT, Hale DS, et al Female pelvic organ prolapse: a comparison of triphasic dynamic MR imaging and triphasic fluoroscopic cystocolopoproctography. Am J Roentgenol 2000;174:81-8.

16. Fielding JR. Practical MR Imaging of Female Pelvic Floor Weakness. RSNA. 2002;22(2):295-304.

17. Regadas FSP, Murad-Regadas SM, Rodrigues LV et al. Anorectal Three-dimensional Endosonography and Anal
Manometry in Assessing Anterior Rectocele in Women. Anew pathogenesis concept and the basic surgical principle. Colorectal Dis 2006 (In press).

\section{Endereço para correspondência:} STHELA MARIA MURAD REGADAS

Av Pontes Vieira $25512^{\circ}$ andar 60130-241 Fortaleza (CE) 